\title{
A GREEDY HEURISTIC FOR LOCATING CROSSOVERS IN CONVEYOR-BASED AMHS IN WAFER FABS
}

\author{
Andrew Johnson \\ 237K Zachry Engineering Center \\ Department of Industrial and Systems Engineering \\ Texas A\&M University \\ College Station, Texas 77843-3131 U.S.A.
}

\author{
Jesus A. Jimenez \\ 601 University Dr. \\ Ingram School of Engineering \\ Texas State University-San Marcos \\ San Marcos, TX 78640-4616, USA
}

\author{
Hector J. Carlo \\ P.O. Box 9043 \\ Department of Industrial Engineering \\ University of Puerto Rico-Mayagüez \\ Mayaguez, PR 00681, USA \\ Dima Nazzal \\ Vernet Lasrado \\ 4000 Central Florida Blvd. \\ Department of Industrial Engineering \\ and Management Systems \\ University of Central Florida \\ Orlando, FL 32816, U.S.A.
}

\begin{abstract}
Finding the optimal layout of Automated Material Handling Systems (AMHS) is critical for the design of next generation semiconductor wafer fabs. This paper proposes a greedy heuristic to determine the configuration of a conveyor-based AMHS featuring turntables and crossovers. Using a conveyor-based analytical model, the heuristic identifies the crossover that provides the greater benefit in terms of work-in process and delivery time reduction. The virtual SEMATECH $300 \mathrm{~mm}$ fab is used to demonstrate the application of the heuristic. Numerical results show that adding crossovers reduced the system's delivery time by up to 22-percent in the scenarios under consideration.
\end{abstract}

\section{INTRODUCTION}

The implementation and operation of an automated material handling system (AMHS) has been identified as one of the critical factors that will affect the transition to 300 Prime and $450 \mathrm{~mm}$ wafer fabs (Temponi et al., 2009). Some recent studies suggest that transfer batches will become smaller in these next generation wafer fabs. In fact, the transfer batch is expected to be 12 wafers per lot in some $300 \mathrm{~mm}$ wafer fabs, replacing the existing 25-wafer lot (Marshal et al. 2008; Bass and Wright 2008). Thus, the AMHS will have to process twice as many moves to maintain the same throughput level. Several wafer manufacturers are evaluating the impact of using conveyor-based AMHSs as the material handling system of $450 \mathrm{~mm}$ wafer fabs (Pettinato and Pillai, 2005). These conveyor-based systems, also referred to as continuous flow transporters (CFT), are expected to provide higher transport capacity and larger local buffering of material at the processing tool level than vehiclebased AMHS. Delivery times (DT) are expected to be shorter and more predictable, which will then result in improved wafer cycle time, higher tool utilizations, and lower work-in process (WIP).

CFT-based analytical models have been recently proposed in Nazzal et al. (2009). These analytical models enable the performance evaluation of a CFT (in terms of expected WIP and DT) at computational speeds that are significantly lower than those obtained by existing simulation methods. The application of these analytical methods allows the exploration of a larger solution space when making decisions concerning the AMHS configuration. With this in mind, the goal of this paper is to integrate an analytical model of a CFT with a heuristic procedure to determine the optimal location of crossovers in a spine fab layout.

Crossovers decrease the wafer lot's travel distance, thus reducing the AMHS's WIP and DT. However, we note that some crossovers will provide greater benefits than others. Therefore, a ranking system is used in order to identify the crossover that results in the greatest benefit. The novelty of our approach is that we evaluate the different design alternatives based 


\section{Johnson, Carlo, Jimenez, Nazzal and Lasrado}

on congestion delays due to the added intersection points in addition to the traditional method of evaluating the improvement in delivery times as a result of the reduced travel distances.

This paper is organized as follows. Section 2 provides a literature review summarizing papers on number and location of crossovers. Section 3 explains the CFT analytical model and the greedy heuristics. Section 4 presents an experimental design and summarizes the results. Finally, Section 5 states our conclusions and briefly explains our future work.

\section{LITERATURE REVIEW}

Very few publications address the problem of determining the number and location of crossovers in a conveyor-based AMHS for semiconductor wafer fabs. Peters and Yang (1997) propose a network flow formulation to determine the number and location of crossovers for the interbay transport system in a spine layout fab. Their objective function minimizes the tradeoff between the increase in crossover construction cost and the decrease in material handling costs. Yang et al. (1999) propose a hybrid tabu search-simulated annealing procedure to solve the MIP in Peters and Yang (1997). In Agrawal and Heragu (2006) and Montoya-Torres (2006), various aspects of factory design, such as facility layout, AMHS design, and AMHS operational issues are discussed. Unfortunately, these studies do not consider the congestion created by incorporating shortcuts to the system.

The conveyor-based continuous flow transport (CFT) literature related to wafer fabs has concentrated on simulation models. For a literature review on conveyors models at wafer fabs, as well as a discussion of advantages and disadvantages of using conveyors in wafer fabs, the reader is referred to Nazzal and El-Nashar (2007).

Several publications are concerned with analytical models for general closed-loop conveyors with multiple stations, similar to the ones in CFT. However, most existing analytical models are designed for a specific manufacturing environment; their modeling assumptions do not properly characterize conveyors commonly found in wafer fabs.

Bozer and Hsieh (2005) present an analytical model for closed-loop conveyors that approximates the throughput capacity and expected WIP in the system. Although the model in Bozer and Hsieh (2005) is designed for general manufacturing environments, their assumptions are not unrealistic for wafer fabs using CFTs. The model from Bozer and Hsieh (2005) does not consider turntables, which are typical for CFT systems in wafer fabs. Nazzal et al. (2008) extends the analytical model for closed-loop conveyors in Bozer and Hsieh (2005) by including turntables at the four corners of the conveyor loop to perform rotations in 90-degree turns Nazzal et al. (2008) conclude that their analytical model performs well with acceptable error. Further, the analytical model was able to detect instability caused by an overloaded conveyor or overloaded turntables. Nazzal et al. (2009) extends the analytical model in Nazzal et al. (2008) to include crossovers. The objective of that study is to correctly estimate the expected Work in Process (WIP) on the conveyor, queuing delays due to congestion at intersection points, as well as assessing the conveyor system stability. A four-phase approach is used to estimate the WIP. Experiments were performed by using the SEMATECH virtual $300 \mathrm{~mm}$ wafer fab. The authors conclude that in the (worst) case, where the maximum number of crossovers is used and the traffic on the conveyor is high, the analytical model performs very well with relative errors on average of $4.2 \%$. This study uses the analytical model from Nazzal et al. (2009) to construct a simple heuristic that is helpful in determining a ranking of the best crossovers to incorporate into the CFT system.

\section{LENGTH CONSTRAINTS}

The objective of this study is to develop a methodology that quantifies the impact of adding shortcuts in conveyor-based material handling layouts. Hence, the proposed methodology would be useful for the design of conveyor-based AMHS. In other words, given a closed-loop CFT, this work identifies which shortcuts should be added to improve the performance of AMHS. The criteria for performance in this study are the expected work-in-process (WIP) on the conveyor and the average delivery time (DT). These criteria are a function of input parameters such as the move requirements, the number of crossovers, the conveyor speed, the layout of the stations on the AMHS closed loop track, the turntables delays, and the window size for each lot. This section will describe the proposed methodology for identifying the best shortcuts to be incorporated into a conveyor-based AMHS.

We propose to identify the best set of crossovers to include in a conveyor-based AMHS by sequentially adding single crossovers until the benefit of adding an additional crossover is less than the cost to construct such a crossover. To quantify the benefits, WIP and average delivery times are estimated using the analytical model in Nazzal et al. (2009). This section briefly introduces the four phase analytical model from Nazzal et al. (2009).

\subsection{Phase I - No turntables}

The Phase I approximation is based on the work of Bozer and Hsieh (2005) for the performance of closed-loop conveyors in a general manufacturing setting. Bozer and Hsieh make several assumptions that will be maintained for the models developed here. Namely: 


\section{Johnson, Carlo, Jimenez, Nazzal and Lasrado}

1. Conveyors travel at a constant speed;

2. Move requests follow a Poisson process;

3. No queuing of the loads is possible at loading and unloading stations;

4. The conveyor is continuous;

5. The conveyor can be divided into equal size segments, in our case, each segment holds one lot.

Define $\alpha_{i}$ to be the mean arrival rate of loads to segment $i, V$ as the speed of the conveyor in terms of windows per time unit, and allow $q_{i}$ to denote the probability that segment $i$ is occupied (provided that the conveyor system is stable). The parameter $q_{i}$ can be calculated from the expression $q_{i}=\alpha_{i} / V$. Finally, expected traveling WIP $\left(W I P_{I}\right)$ on the conveyor is estimated using the expression $W I P_{I}=\sum_{i \in S} w_{i} q_{i}$, where $S$ is the number of segments.

\subsection{Phase II and Phase III - Turntables Analysis}

Turntables are analyzed in pairs by considering two turntables located on the same crossover (the end of the intrabay conveyor can also be thought of as crossovers). The downstream turntables do not create queues because turntables have identical and deterministic turning time and lots on crossovers receive priority. The expected WIP at the downstream corner turnta-

bles is simply its utilization, $\lambda_{c} t$. The upstream corner turntable can be analyzed as an $M / D / 1 / b$ system, as in Buzacott and Shanthikumar (1993), where $b$ is the number of windows separating the upstream turntable and the upstream station. The expected WIP $\left(W I P_{I I}\right)$ at the upstream turntable is estimated by:

$$
W I P_{I I}=\left(\frac{1}{2}\right)\left(\frac{\lambda_{c}^{2} t^{2}}{1-\lambda_{c} t}\right)+\lambda_{c} t
$$

where $\lambda_{c}$ is the arrival rate of lots to the corner turntable, and $t$ is the turning time of the turntable. The stability conditions necessary to apply the above formula are described in Nazzal et al. (2008).

Next the waiting delays created by the addition of crossovers are considered. A cell, shown in Figure 1, is defined by four stockers, labeled $p, q, r$, and $s$. It is possible to consider the insertion of two crossovers in a cell. The inclusion of four turntables, labeled $e, f, g$, and $h$ below, is necessary to access the crossovers.

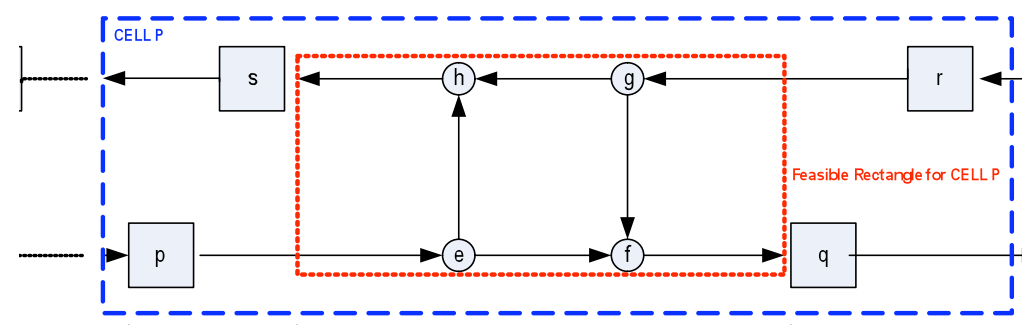

Figure 1: Cell $p$ consists of two crossovers in opposite directions

Let, $T_{e}, T_{g}, T_{f}$, and $T_{h}$ be the expected delay at the queues that form due to turntables $e, g, f$, and $h$, respectively. Arrival rates of lots to crossovers, $\lambda_{e h}$ and $\lambda_{g}$, shown below, are estimated as the average number of lots per time that will require travel on, respectively, crossovers $e h$ and $g f$ to take the shortest distance path from their origin stocker to their destination stocker.

$$
\begin{aligned}
& \lambda_{e h}=\sum_{i=s+1}^{p} \sum_{j=s}^{i-1} \alpha_{i j}-\sum_{k l \in U_{e h}} \lambda_{k l} y_{k l} \\
& \lambda_{g f}=\sum_{i=q+1}^{r} \sum_{j=q}^{i-1} \alpha_{i j}-\sum_{k l \in U_{g f}} \lambda_{k l} y_{k l}
\end{aligned}
$$

where $\alpha_{i j}$ is the average rate of lots traveling from stocker $i$ to stocker $j$, stocker $N+1$ is stocker 1 , and, and $U_{g f}\left(U_{e h}\right)$ is the set of crossovers upstream of crossovers $g f(e h)$ and in the same direction. $y_{k l}$ is an indicator variable that crossover $k l$ is installed $\left(y_{k l}=1\right)$, or not $\left(y_{k l}=0\right)$. The assumptions and directions for implementation of this set of equations is presented in Nazzal et al. (2009). The mean arrival rates of loads to segments between crossovers within a cell $\lambda_{e f}$ and $\lambda_{g h}$ are estimated as 
the average number of lots per time that will travel from stockers $s, s+1, \ldots, p$ to stockers $q, q+1, \ldots, r$, plus -if the prior shortcut was not installed- the lots that would have traveled on the prior crossover. These are stated as

$$
\begin{aligned}
& \lambda_{e f}=\sum_{i=s}^{p} \sum_{j=q}^{r} \alpha_{i j}+\lambda_{g f}\left(1-y_{g f}\right) \\
& \lambda_{g h}=\sum_{i=q}^{r} \sum_{j=s}^{p} \alpha_{i j}+\lambda_{e h}\left(1-y_{e h}\right)
\end{aligned}
$$

The average delays at input turntable of cell (e or g) can be estimated via an M/D/1 queue with arrival rate $\lambda_{p e}=\lambda_{e h}+\lambda_{e f}$ or $\lambda_{r g}=\lambda_{g f}+\lambda_{g h}$, respectively. The expected service time can be estimated as $t$ for a turning load and 0 for a passing load; where $t$ is the time required by the turntable to rotate the load 90 degrees, wait for the load to get off the turntable, and turn back 90 degrees to its original position. By the Pollaczek-Khintchine formula, the expected WIP, $L_{e}, L_{g}, L_{f}$, and $L_{h}$, due to turntables $e, g, f$ and $h$, respectively, are:

$$
\begin{gathered}
L_{e}=\frac{\lambda_{p e} \lambda_{e h} t^{2}}{2\left(1-\lambda_{e h} t\right)}+\lambda_{e h} t \\
L_{g}=\frac{\lambda_{r g} \lambda_{g f} t^{2}}{2\left(1-\lambda_{g f} t\right)}+\lambda_{g f} t \\
L_{f}=\frac{\lambda_{g f}^{2} t^{2}}{2}+\lambda_{g f} t \\
L_{h}=\frac{\lambda_{e h}^{2} t^{2}}{2}+\lambda_{e h} t
\end{gathered}
$$

The equivalent equations for the expected delays due to turntables can be calculated by applying Little's Law to the formulas above. For details on the derivation of Equations (1)-(8) and the equations for expected delays due to turntables the reader is referred to Nazzal et al. (2009).

\subsection{Phase IV: Estimating Total Expected WIP}

The expected WIP on the conveyor can be estimated as:

$$
W I P_{\text {conv }}=\underbrace{\sum_{i \in S} w_{i} q_{i}}_{\begin{array}{l}
\text { Phase I: } \\
\text { travelling WIP }
\end{array}}+\underbrace{\left(\frac{1}{2}\right)\left(\frac{\lambda_{l c}^{2} t^{2}}{1-\lambda_{l c} t}\right)+2 \lambda_{l c} t}_{\begin{array}{c}
\text { Phase II: } \\
\text { two right corner turntables }
\end{array}\left(\frac{1}{2}\right)\left(\frac{\lambda_{r c}^{2} t^{2}}{1-\lambda_{r c} t}\right)+2 \lambda_{r c} t}+\underbrace{\underbrace{}_{\text {accumulated WIP due to turntables } e, g, f, h \text { in each cell }}}_{\begin{array}{c}
\text { Phase II: } \\
\text { two left corner } \\
\text { turntables }
\end{array}}
$$

The first term is the traveling WIP on every segment $i$ in the conveyor, $i \in S, S$ is the set of segments in the conveyor network, summing all the estimated WIP traveling on each window $w_{i}$. The second and third terms are the expected WIP accumulating due to the right and left pairs of corner turntables (subscripts $r c$ and $l c$ refer to right and left corner, respectively). The forth term is the accumulating $W I P$ due to the crossover, the detailed expressions for $L_{e}, L_{g}, L_{f}$, and $L_{h}$ are given in Equations (5)-(8). The term $y_{i}$ is the indicator $(0,1)$ variable activated if crossover $i$ is installed.

\subsection{Model Validation}

A simulation model of a virtual fab developed by International SEMATECH was used in Nazzal et al. (2009) for validating the analytical model. A numerical study was conducted to evaluate the analytical model over a wide range of operating scenarios. The authors investigated the impact of several factors: structure of the from-to matrix, coefficient of variation of move requests arrival process, the volume of move requests, and the speed of the conveyor and turntable delay. Validation was performed for the average delays at each turntable and for the overall work-in-process on the conveyor given in Equation (9). The relative error in estimating the turntable delays under extreme experimental conditions averaged less than $8 \%$ and the relative error in estimating the expected work-in-process on the conveyor was less than $7 \%$. Details of the validation numerical study can be found in Nazzal et al. (2009). 


\subsection{A Heuristic to Identifying the Next Crossover for Inclusion}

Using the formulas presented above the performance of a specified layout can be estimated. However, for large wafer fabs complete enumeration of all potential crossover combinations is not practical as there are $2^{\mathrm{n}}$ possibilities (where $n$ is the number of potential crossovers). The following greedy heuristic (H1) is proposed for determining the set of crossovers to include.

Step 1) Start with a conveyor system without any crossovers.

Step 2) Using Equation 9, evaluate the effects of adding each crossover independently. Rank the crossovers according to their impact identify the best crossover to add to the system. The ranking of crossovers is determined as the relative improvement in WIP comparing a layout with no crossovers to a layout with only the crossover under consideration included.

Step 3) Identify the highest ranked remaining crossover. If the marginal impact of adding the crossover exceeds the cost of constructing such a crossover, go to Step 4; otherwise STOP as the heuristic procedure has terminated.

Step 4) The heuristic recommends adding this crossover. Eliminate the crossover from the ranking and return to Step 3.

It is important to highlight that the proposed heuristic ignores the interdependency of crossovers. In other words, we are ignoring the effects of adding a crossover upstream could have on a crossover downstream.

\section{COMPUTATIONAL EXPERIMENTS}

In this section, the proposed heuristic is tested to decide on the best set of locations for the crossovers using the SEMATECH layout illustrated in Figure 2. This is the layout for the interbay system with 24 stockers supplying each of the 24 functional bays. The labeled arcs represent a crossover with $i_{\mathrm{U}}$ denoting shortcut $i$ in the upward direction and $i_{\mathrm{D}}$ denoting shortcut $i$ in the downward direction. (Notice that there are 22 possible crossovers in this layout. Hence, $\mathrm{H} 1$ will require 44 evaluations while $\mathrm{H} 2$ will require $1 \times 10^{21}$ evaluations.)

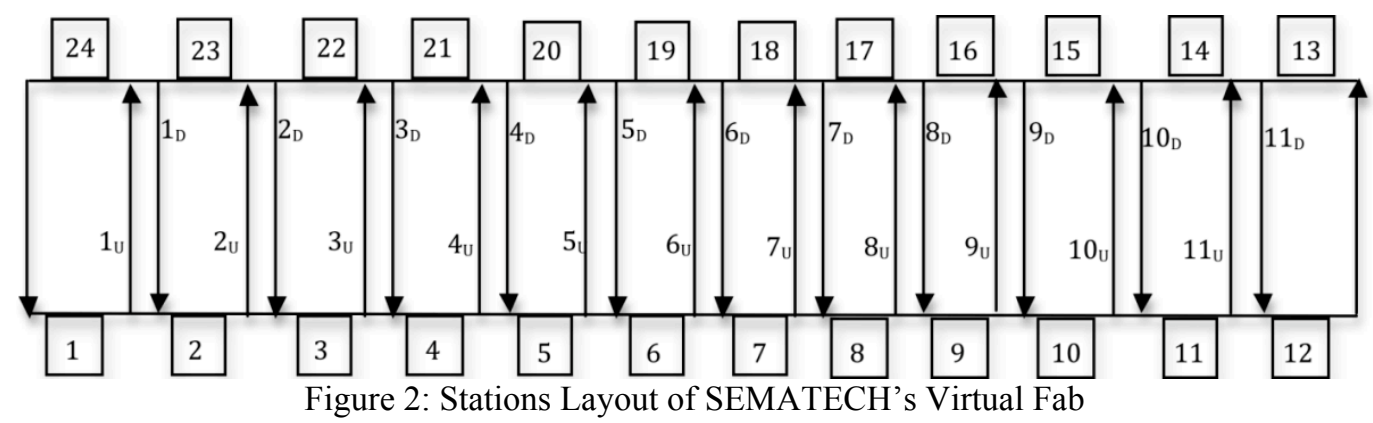

The virtual fab model developed by International SEMATECH is used to decide on the best subset of crossovers to add to the conveyor network so as to minimize the expected work-in-process on the conveyor and the average delivery time of lots. The SEMATECH model is a representation of a $300 \mathrm{~mm}$ wafer fab; the $300 \mathrm{~mm}$ Wafer Fab Model has 24 bays arranged using a spine layout configuration. In the original model, each bay has two stockers and can accommodate up to 24 tools, for simplification, we assume that each bay will be served by one stocker. For the purpose of this design study, we modified some parameters of the original $300 \mathrm{~mm}$ Wafer Fab Model to create additional scenarios for testing the design heuristic. These modifications created the operating scenarios specified in Table 1. The factors considered in the validation study include the Fab bottleneck processing tool capacity (Factor A), Move Request Rates (Factor B), and the ratio of the Conveyor Speed to the Turntable Speed (Factor C).

The description for each factor is summarized as follows:

A. Fab bottleneck capacity: This factor represents the utilization of the bottleneck tool in the fab. It is a function of the release rate of wafer lots to the fab, and is found by changing the average release rate of wafer lots, and noting the bottleneck tool utilization. The two capacity levels studied are $80 \%$ and $97 \%$ utilizations, with a corresponding number of moves of 232 moves per hour and 290 moves per hour, respectively.

B. Expected Rate of Move Requests: This factor indicates the frequency of moves between a pair of stockers. Three different From-To matrices are considered, level 1 has the same from-to matrix as SEMATECH process flow, level 2 is similar to level 1 with heavier traffic requiring longer travel distances, and level 3 is an extreme scenario where the move requests are uniformly distributed among all stockers (i.e. every stocker sends the same number of lots to every other stocker). 
C. Conveyor Speed/Turntable Cycle Time: This factor is a combination of two conveyor settings; the conveyor speed and turntable turning time. Two cases are considered, fast and slow. For the slow case, the conveyor speed is $0.305 \mathrm{ft} / \mathrm{s}$ and the turntable cycle time is $15 \mathrm{sec}$. For the fast case, the conveyor speed is $1.000 \mathrm{ft} / \mathrm{s}$ and the turntable cycle time is $5 \mathrm{sec}$.

Table 1: Factors considered in the experiments and their levels

\begin{tabular}{clccc} 
Factor & \multicolumn{1}{c}{ Name } & Level 1 & Level 2 & Level 3 \\
\hline A & Bottleneck Capacity & $80 \%$ & $97 \%$ & - \\
B & Move Request Rates & SEMATECH & SEMATECH & Uniform \\
& & Flow & Constrained & Flow \\
C & Conveyor Speed & Slow & Fast & - \\
& /Turntable Cycle Time & & & \\
\hline
\end{tabular}

\subsection{Results}

Table 2 shows the average delivery time and expected WIP on the conveyor for each combination of the factors listed in Table 1. These results are based on having no crossovers and thus the conveyor operates as a simple rectangular loop with turntables at the four corners.

Table 2: No crossovers average WIP and delivery time at each factors combination

\begin{tabular}{lcc}
\hline & $\begin{array}{c}\text { Average } \\
\text { Delivery Time } \\
\text { (sec.) }\end{array}$ & $\begin{array}{c}\text { Average } \\
\text { WIP } \\
\text { (lots) }\end{array}$ \\
\hline A: 80\%, B: SEMATECH Flow, C: Slow & 1638 & 106 \\
A: 80\%, B: SEMATECH Flow, C: Fast & 499 & 32 \\
A: 97\%, B: SEMATECH Flow, C: Slow & 1648 & 133 \\
A: 97\%, B: SEMATECH Flow, C: Fast & 500 & 40 \\
A: 80\%, B: SEMATECH Constrained, C: Slow & 1582 & 168 \\
A: 80\%, B: SEMATECH Constrained, C: Fast & 476 & 50 \\
A: 97\%, B: SEMATECH Constrained, C: Slow & 1738 & 222 \\
A: 97\%, B: SEMATECH Constrained, C: Fast & 479 & 61 \\
A: 80\%, B: Uniform Flow, C: Slow & 1569 & 102 \\
A: 80\%, B: Uniform Flow, C: Fast & 478 & 31 \\
A: 97\%, B: Uniform Flow, C: Slow & 1574 & 127 \\
A: 97\%, B: Uniform Flow, C:Fast & 478 & 39 \\
\hline
\end{tabular}

The proposed greedy heuristic was applied for each of the combinations listed in Table 1. In this study, we decided that the stopping rule for the heuristic was when the reduction in the average delivery time is less than $5 \%$. Figures $3-5$ illustrate the ranking of the shortcuts for each level of the average flows factor B, Tables 3-5 show the average delivery time and average WIP associated with adding each crossover independently as well as the reduction in the delivery time as a result of adding that crossover.

Table 3: Ranking of crossovers for the SEMATECH-based flows (80\% bottleneck capacity; slow conveyor setting)

\begin{tabular}{lccc}
\hline Crossover & Average WIP & Average Delivery Time (sec.) & \% Reduction \\
\hline 7U & 84 & 1297 & $21 \%$ \\
8U & 85 & 1318 & $20 \%$ \\
9U & 88 & 1364 & $17 \%$ \\
6U & 89 & 1369 & $16 \%$ \\
5U & 92 & 1415 & $14 \%$ \\
10U & 92 & 1422 & $13 \%$ \\
3D & 93 & 1443 & $12 \%$ \\
2D & 94 & 1452 & $11 \%$ \\
4D & 95 & 1461 & $11 \%$ \\
4U & 96 & 1478 & $10 \%$ \\
11U & 97 & 1503 & $8 \%$ \\
5D & 97 & 1504 & $8 \%$ \\
6D & 99 & 1537 & $6 \%$ \\
1D & 100 & 1539 & $6 \%$ \\
\hline
\end{tabular}




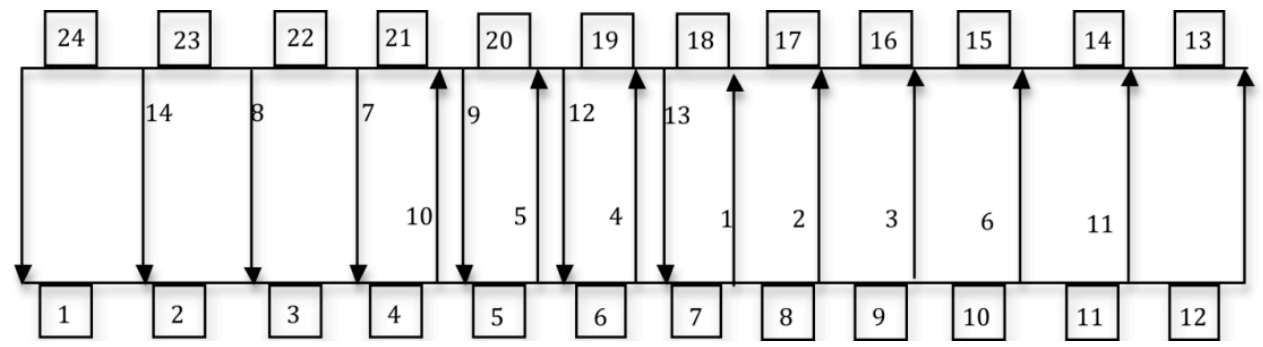

Figure 3: Ranking of crossovers for SEMATECH-based flows (80\% bottleneck capacity; slow conveyor setting)

Table 4: Ranking of crossovers for the SEMATECH-constrained flows (80\% bottleneck capacity; slow conveyor setting)

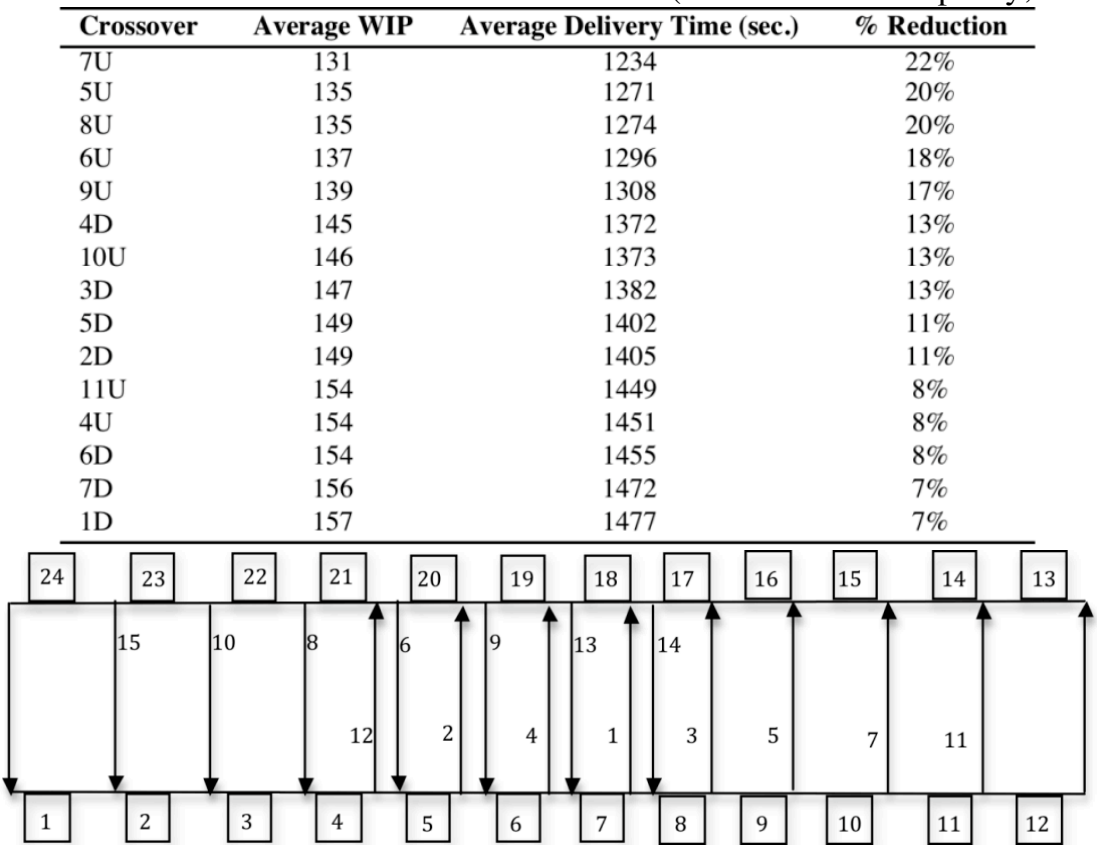

Figure 4: Ranking of crossovers for SEMATECH Constrained flows (80\% capacity; slow conveyor setting).

Table 5: Highest ranked crossovers for the SEMATECH-uniform flows (80\%bottleneck capacity; slow conveyor setting)

\begin{tabular}{lccc}
\hline Crossover & Average WIP & Average Delivery Time (sec.) & \% Reduction \\
\hline $8 \mathrm{U}$ & 87 & 1339 & $15 \%$ \\
$7 \mathrm{U}$ & 87 & 1350 & $14 \%$ \\
9U & 88 & 1354 & $14 \%$ \\
4D & 88 & 1354 & $14 \%$ \\
5D & 88 & 1364 & $13 \%$ \\
3D & 88 & 1366 & $13 \%$ \\
6U & 89 & 1377 & $12 \%$ \\
10U & 90 & 1385 & $12 \%$ \\
6D & 90 & 1390 & $11 \%$ \\
2D & 91 & 1399 & $11 \%$ \\
5U & 92 & 1417 & $10 \%$ \\
7D & 92 & 1424 & $9 \%$ \\
11U & 94 & 1448 & $8 \%$ \\
4U & 95 & 1462 & $7 \%$ \\
8D & 95 & 1465 & $7 \%$ \\
1D & 95 & 1472 & $6 \%$ \\
\hline
\end{tabular}




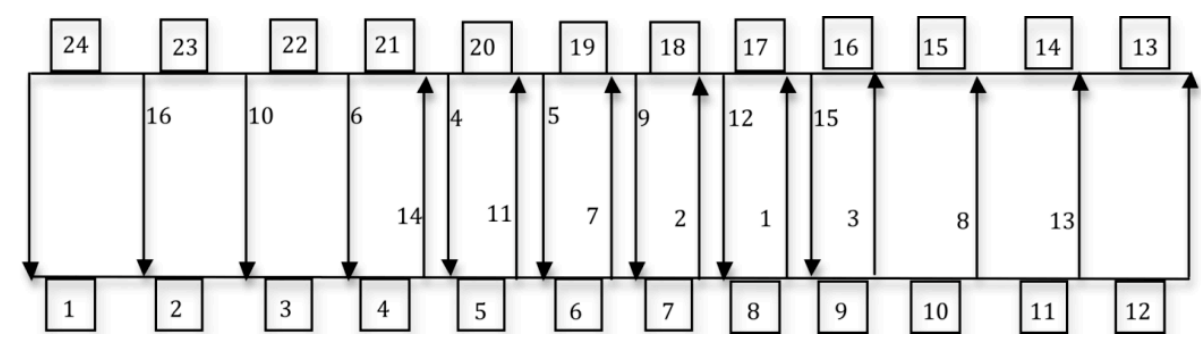

Figure 5: Ranking of crossovers for Uniform flows (80\% capacity; slow conveyor setting).

Table 6 shows the percent reduction in the average delivery times after adding all the identified crossovers illustrated in Figures 3-5.

Table 6: Improvement in average delivery time after adding the highest ranked crossovers

\begin{tabular}{lccc}
\hline & $\begin{array}{c}\text { No crossovers } \\
\text { average } \\
\text { delivery time } \\
\text { (sec.) }\end{array}$ & $\begin{array}{c}\text { Number of } \\
\text { crossovers } \\
\text { added }\end{array}$ & $\begin{array}{c}\text { \%eduction } \\
\text { in delivery } \\
\text { time }\end{array}$ \\
\hline A: $80 \%$, B: SEMATECH Flow, C: Slow & 1638 & 14 & $56.3 \%$ \\
A: $80 \%$, : SEMATECH Constrained, C: Slow & 1582 & 15 & $60.5 \%$ \\
A: $80 \%$, B: Uniform Flow, C: Slow & 1569 & 16 & $55.8 \%$ \\
\hline
\end{tabular}

\subsection{Discussion of the Results}

The results we presented in the tables and figures above are only displaying the rankings for one setting of factors A and C: $80 \%$ bottleneck utilization and slow conveyor setting, respectively. The reason the other levels were omitted is because no significant difference in the ranking of the crossovers was observed at higher traffic rate or when the conveyor is faster and the turning time of turntables is shorter. The only factor that had an impact on the preferred location of the crossovers is the flow rate matrix structure.

Adding crossovers is always beneficial for reducing the average WIP and delivery time even when we take into consideration the queuing delays introduced by adding the shortcuts. This is expected because with no crossovers, the lots that would have used the crossover are routed using the longest distance passing through the corner turntables. Adding crossovers is equivalent to moving from a one server one queue waiting line to a multi-server, multi-queues system and so naturally the expected WIP never increases when we add a crossover. This discussion is based on the assumption that the turntables of the crossovers do not interfere with each other, nor with the stations that drop-off the loads onto the conveyor. If interference is an issue, the turntables might block one another and the long queue of a turntable might extend to block the upstream turntable or the upstream station resulting in a different behavior from what we saw here in our experiments.

Examining the uniform flows scenario; we would expect that the best locations of crossovers should be the ones in the middle of the loop. However, in our implementation this was not necessarily the case, as we can see in Figure 5 . The crossovers in the middle, denoted by $6_{U}$ and $6_{D}$ are ranked $7^{\text {th }}$ and $9^{\text {th }}$ respectively. The reason is that only one crossover from each cell is being considered in each iteration rather than the pair of crossovers in opposite directions occupying the same location, also referred to earlier as a "cell". If we run the same heuristic but instead of considering adding a crossover in each iteration, we evaluate the benefit of adding each cell independently, we would get the intuitive result of crossovers in the middle being the most attractive, as we see in Figure 6. The slight preference of the crossovers in the right half to those in the left half of the loop is due to lack of perfect symmetry in the layout.

Finally, this heuristic provides a ranking of the crossovers independently, and therefore, we are ignoring the benefit of adding a crossover given that another crossover has already been added. Considering the impact of adding a crossover on the conveyor performance given the already added crossovers is one way we can improve the heuristic and while it would require more computational time it would still be tractable. 


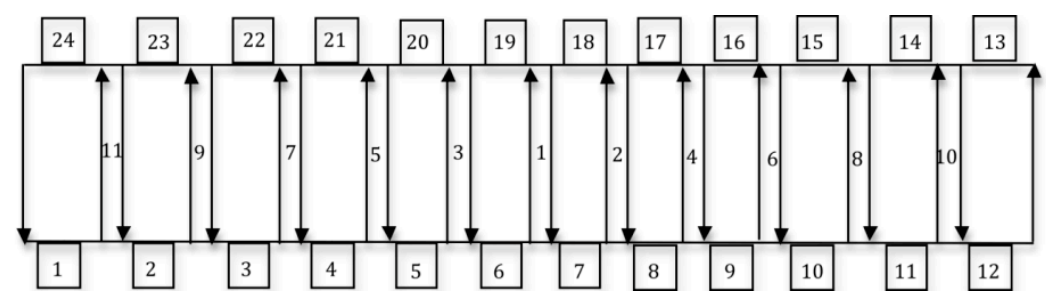

Figure 6: Ranking of crossovers for SEMATECH-uniform flows by adding one cell at a time.

\section{CONCLUSIONS}

This paper introduces a heuristic to identify a set of crossovers to include in a conveyor-based material handling system within a wafer fab. The heuristic is greedy in the sense that a sequential set of decisions is made adding individual crossovers to improve the performance of the material handling system. The performance of the heuristic is analyzed for the $300 \mathrm{~mm}$ virtual fab model developed by International SEMATECH. The results indicate that the inclusions of crossovers can have a significant effect on average delivery time and WIP. Further, experimental results indicate that the speed of the conveyor or the volume of move requirements has no effect on the ordering of crossovers to consider for inclusion. Rather, the only important factor is the distribution of move requests among bays. This paper is a continuation of a line of research with the purpose to quantify the relative advantages and disadvantages of conveyor-based material handling systems relative to the more traditional vehicle-based systems. A relatively strong assumption has been made in the current paper that the effects of crossovers are independent. As part of our future research, the research teams hopes to relax this assumption and identify the set of crossovers that has the largest impact on the performance of the material handling system in this more general setting.

\section{REFERENCES}

Agrawal, G.K., and S.S. Heragu. 2006. A survey of automated material handling systems in 300-mm Semiconductor Fabs. IEEE Transactions on Semiconductor Manufacturing 19:112-120.

Bass, E. and R. Wright. 2008. Modeling semiconductor factories for equipment and cycle time reduction opportunities, $F u$ ture Fab International 24:50-55.

Bozer, Y.A., and Y-J. Hsieh. 2005. Throughput performance analysis and machine layout for discrete-space closed-loop conveyors. IIE Transactions 37:77-89.

Marshall, L., K. Rust, and K. Schmidt. 2008. 300-mm Prime Gaps That Need to Be Addressed to Boost Productivity. IEEE Transactions on, Semiconductor Manufacturing 21:592-599.

Montoya-Torres, J.R. 2006. A Literature Survey on the Design Approaches and Operational Issues of Automated WaferTransport Systems for Wafer Fabs. Production Planning and Control 7:648-663.

Nazzal, D., and A. El-Nashar. 2007. Survey of research in modeling conveyor-based automated material handling systems in wafer fabs. In Proceedings of the 2007 Winter Simulation Conference, eds. S. G. Henderson, B. Biller, M.-H Hsieh, J. Shortle, J. D. Tew, and R. R. Barton, 1977-1985. Piscataway, New Jersey: Institute of Electrical and Electronics Engineers, Inc., 1760-1767.

Nazzal, D., A. Johnson, H.J. Carlo, and J.A. Jimenez. 2008. An analytical model for conveyor based AMHS in semiconductor wafer fabs. In Proceedings of the 2008 Winter Simulation Conference, eds. S. J. Mason, R. R. Hill, L. Mönch, O. Rose, T. Jefferson, J. W. Fowler, 463- 470. Piscataway, New Jersey: Institute of Electrical and Electronics Engineers, Inc., 2148-2155.

Nazzal, D., J.A. Jimenez, H.J. Carlo, A. Johnson, and V. Lasrado. 2009. An analytical model for conveyor based automated material Handling system with crossovers in semiconductor wafer fabs. Working paper.

Peters, B.A., and T. Yang. 1997. Integrated facility layout and material handling system design in semiconductor fabrication facilities. IEEE Transactions on Semiconductor Manufacturing 10:360-369.

Pettinato, J.S. and D. Pillai. 2005. Technology decisions to minimize 450-mm wafer size transition risk. IEEE Transactions on Semiconductor Manufacturing 18: 501-509.

Temponi, C., J.A. Jimenez, and J. Khan. 2009. Preliminaries on AMHS: Lessons learned. In Proceedings of the 2009 International Conference on Industrial Engineering and Systems Management, May 13-15.

Yang, T., M. Rajasekharan, and B.A. Peters. 1999. Semiconductor fabrication facility design using a hybrid search methodology. Computers \& Industrial Engineering 36:565-583. 


\section{AUTHOR BIOGRAPHIES}

ANDREW L JOHNSON is an Assistant Professor in the Department of Industrial and Systems Engineering at Texas A\&M University. He obtained his M.S. and Ph.D. in The H. Milton Stewart School of Industrial and Systems Engineering from Georgia Tech, and his B.S. in Industrial and Systems Engineering from Virginia Tech. His research interests include productivity and efficiency measurement, microeconomics, warehouse design and operations, material handling and Internet based database applications. He is a member of the Institute of Industrial Engineers, INFORMS, National Eagle Scout Association, and German Club of Virginia Tech. His email address is $<a j o h n s o n @ t a m u . e d u\rangle$.

HECTOR J. CARLO is an Assistant Professor in the Industrial Engineering Department at the University of Puerto Rico Mayagüez. He graduated with a BS in Industrial Engineering from the University of Puerto Rico - Mayagüez (2001), and a MSE (2003) and PhD (2007) from The University of Michigan in Industrial and Operations Engineering. His current research interests include the design and optimization of automated material handling systems, container terminals, cross-docks, and warehouses, as well as Reconfigurable Manufacturing Systems. He is a registered Professional Engineer in the commonwealth of Puerto Rico. His email address is $\langle$ hector.carlo@upr.edu>.

JESUS A. JIMENEZ is an Assistant Professor in the Industrial Engineering program of the Ingram School of Engineering at Texas State University -San Marcos. He obtained his Ph.D. in Industrial Engineering from Arizona State University, and his M.S. and B.S. in Industrial Engineering from The University of Texas at El Paso. His research interests are in the modeling and analysis of manufacturing systems, especially semiconductor manufacturing; automated material handling systems; the development of manufacturing applications of operations research, computer simulation, and statistics; and the reduction of time required to build, experiment, and analyze complex computer simulation models. His email address is <jesus.jimenez@txstate.edu>.

DIMA NAZZAL is an Assistant Professor at the department of Industrial Engineering and Management Systems at the University of Central Florida, Orlando, Florida. She obtained her Ph.D. degree from The H. Milton Stewart School of Industrial and Systems Engineering at Georgia Tech, Atlanta, Georgia, in 2006, and her M.S. in Industrial Engineering from The University of Central Florida. She earned her B.S. in Industrial Engineering from The University of Jordan. She is a member of INFORMS and IIE. Her research interests are in analytical modeling of manufacturing and logistics systems. She can be contacted by email at < dnazzal@mail.ucf.edu>.

VERNET LASRADO is a Ph.D. student in the Department of Industrial Engineering and Management Systems at the University of Central Florida. He received his Bachelors degree from the Georgia Institute of Technology. He can be contacted by email at <vernet.lasrado@gmail.com>. 